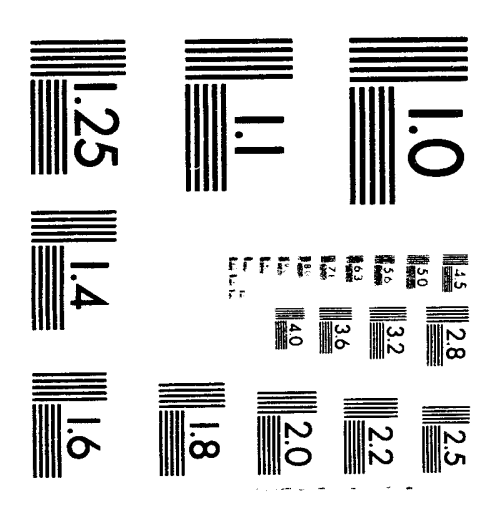



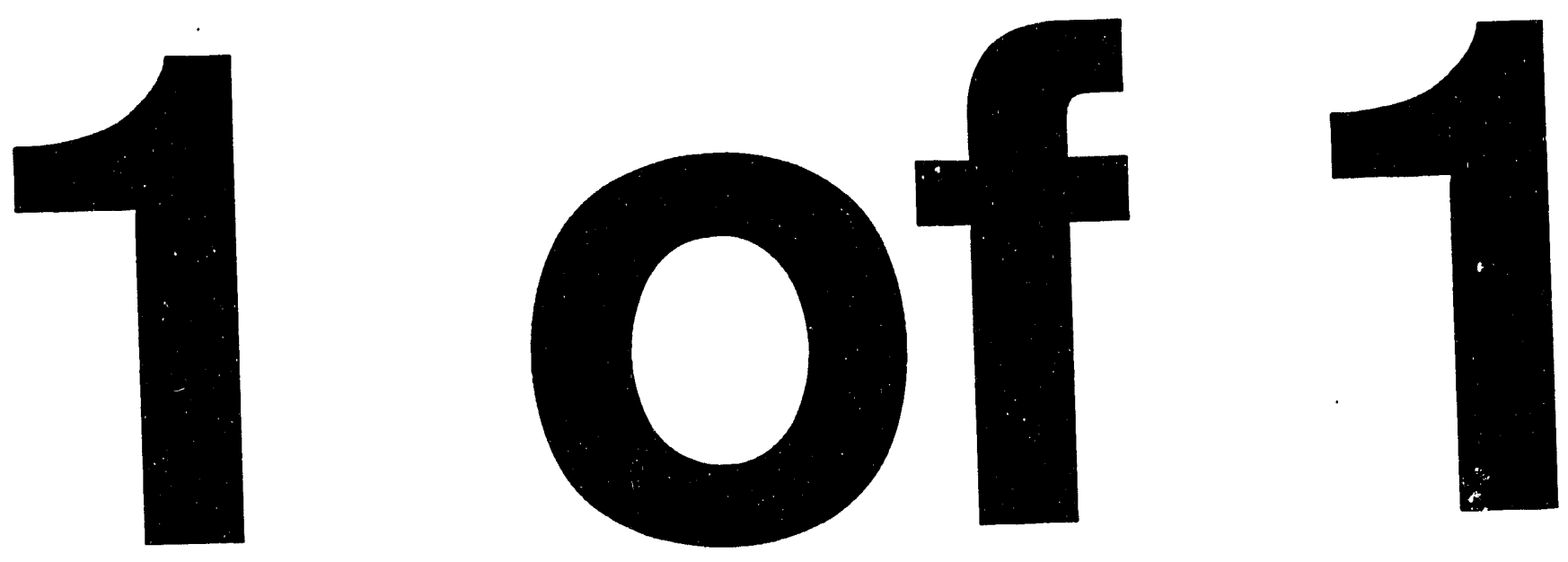


\title{
NMR IMAGING OF COMPONENTS AND MATERIALS FOR DOE APPLICATION
}

\author{
Bonny R. Richardson
}

DECEMBER 1993

\section{Production Capability Assurance Program Current Issues \\ PX-93-3-019}

Keywords:

Nuclear Magnetic Resonance Imaging

NMRI

MRI

Solid State

Explosives

Polymers

Elastomers 


\title{
NMR IMAGING OF COMPONENTS AND MATERIALS FOR DOE APPLICATIONS
}

\author{
Benny R. Richardson
}

\author{
DECEMBER 1993
}

\section{Production Capability Assurance Program \\ Current Issues \\ PX-93-3-019}

\begin{abstract}
The suitability for using NMR imaging to characterize liquid, polymeric, and solid materials was reviewed. The most attractive applications for NMR imaging appear to be liquid-filled porous samples, partially cured polymers, adhesives, and potting compounds, and composite polymers/high explosives containing components with widely varying thermal properties. Solid-state NMR line-narrowing and signal-enhancing markedly improve the imaging possibilities of true solid materials. These techniques provide unique elemental and chemical shift information for highly complex materials and complement images with similar spatial resolution, such as $\mathrm{X}$-ray computed tomography (CT).
\end{abstract}




\section{INTRODUCTION}

Magnetic Resonance Imaging (MRI), more properly termed Nuclear Magnetic Resonance Imaging (NMRI), has become a standard imaging method for examining biological samples and some non-biological polymers. The clarity of images rivals those of standard $X$-ray computed tomography (CT), and actually surpasses $X$-ray image clarity for soft tissues with poor $X$-ray contrast. Industrial applications of NMRI are multiplying, and require both conventionally developed pulse programs and novel, high-power methods to image solids. This paper serves as a review of the many NMRI methods to cull those found most useful for imaging many of the complex materials used for DOE applications. The review is not intended to be exhaustive, but rather selective in its discussion of methods most relevant to materials used within the DOE weapons complex. For instance, rotating-frame magic-angle imaging and field modulating, selective spin imaging will not be discussed. More extensive reviews are available for conventional "liquid-like" imaging(1) and for solid-state imaging.(2)

The attractiveness of NMRI for our uses is many-fold. Unlike other imaging radiation, NMRI is non-destructive and non-invasive to the sample. NMRI uses megahertz $(\mathrm{mHz})$ frequency electromagnetic radiation (radio waves) for examining the sample, not $X$-rays. The use of NMRI might replace some present $X$-ray imaging applications and minimize personnel exposure to $X$-rays. The minimization of personnel exposure to $X$-ray flux is consistent with DOE's aslow-as-reasonably-achievable (ALARA) goals for high energy radiation exposure. Further attractions deal with the elimination of various waste streams from $X$-ray film development and the reduction in the number of processed and stored $X$-ray films. The latter is an important point, given the expense of film and the thousands of films which require storage.

Conventional $X$-ray imaging is performed at the Pantex Plant to detect internal parts, foreign inclusions, voids, and cracks in weapon components. Image contrast comes from differences in absorption of $X$-rays by the material being imaged. One can reasonably expect spatial resolution of $25 \mu \mathrm{m}$ from a standard $X$-ray image. Any alternate technique must approach this limit. Moreover, the method must be able to examine samples ranging in size from $1-\mathrm{cm}$ to many centimeter diameter (and of varying thickness). The method must be reasonably quick to avoid backlogging production/disassembly schedules. If a spatial resolution of tens of $\mu \mathrm{m}$ can be achieved with NMRI, then the method will be of value. Hopefully, the discussion below will reveal if NMRI can reasonably be pushed to achieve these limits.

\section{BASIC PRINCIPLES}

The resonance frequency of a half-integral spin nucleus depends upon the strength of the $B_{\text {local }}$ field, the major component of which is an extremely stable, externally applied magnetic field $B_{0}$. Variations in the $B_{\text {local }}$ field with nuclei in different electronic environments provides for the number of peaks (and the beauty) of an NMR spectrum. All of these peaks, however, are found at frequencies within only parts per million of the nuclei's resonance frequency as calculated from $B_{0}$. In some samples, such as a biological sample containing cell/liquid differentiation, NMR-active nuclei are regionally restricted in position. The vast majority of imaging is ${ }^{1} \mathrm{H}$ imaging due to its favorable abundance and NMR relaxation parameters. The relative positions of protons within a sample can be coded by imposing magnetic field 
gradients across the sample. The degree of spatial resolution of the resulting image can be expressed as a function of the natural linewidth of the protons:

$$
(\Delta x)(\gamma)(G)>\delta \nu_{1 / k}
$$

In this equation, $\Delta x$ stands for spatial resolution, $\gamma$ for the magnetogyric ratio of ' $H, G$ for the strength of the applied gradient, and $\delta \nu_{y_{z}}$ for the linewidth at half-maximum peak height. Linewidth is a function of $T_{2}$, the time constant characterizing spin-spin relaxation. $T_{2}$ is always less than or equal to $T_{1}$, the time constant characterizing spin-lattice relaxation. $T$, is a function of the spectral density of local magnetic fields fluctuating at the nuclei's resonance frequency; the key property, therefore, of samples that restricts which NMRI method is used is molecular motion that produces fluctuating magnetic fields. For instance, samples that are mostly liquid or liquid-like, such as water, can be imaged with conventional medical instrumentation and pulse programming because of the rapid motion of liquid water.

The relaxation mechanisms prevalent in liquids serve to insure that linewidths of 1 to $10 \mathrm{~Hz}$ are maintained, and high resolution images are obtained. Different types of liquid imaging are known(1), and NMR images have been developed by General Electric Company, Phillips, and others.

At the molecular motion extreme of solid rigid samples, the insignificant fraction of magnetic fields fluctuating at the resonance frequency and the presence of chemical shift anisotropy serve to yield very broad lines. From equation(1), much higher magnetic field gradients are necessary to acquire images of the same spatial resolution. To compensate for this extreme situation, solid-state line-narrowing techniques such as high-power decoupling for heteronuclear dipolar interaction(3), coherent line-narrowing pulse sequences for proton-proton dipolar interaction(4), and magic-angle spinning for the chemical shift anisotropy(5) are used separately or jointly to minimize the natural linewidths and the magnitude of gradients required to obtain an image. Solids and composites are most frequently imaged with NMR spectrometers to obtain the highest possible field strengths. In many instances, spectrometer usage and the number of mathematical functions which must be performed per sample unit volume limit the size of sample which can be imaged.

Lying between the extremes of mostly water samples and solid samples are a wide variety of polymers, composites, foams, and other porous samples which may contain liquid or liquidlike components. Some of these complex materials have components that acquire liquid-like characteristics at temperatures higher than ambient, and in that way resemble the plasticbonded explosives (PBX) used in the weapon complex. Silicone potting compounds, adhesives, plastic sleeve material, and other plastic weapon components might be candidates for NMR imaging. Studies of solvent penetration into plastics $(6)$ can provide the basis for long-term compatibility between weapon components and cleaning agents. Studies of paste explosives could detect heterogeneity in mixing and component separation. Indeed, the wide breadth of possible applications within the DOE complex actually drove this review of NMR imaging. 


\section{NMRI CHARACTERIZATION OF LIQUID-LIKE NONMEDICAL SAMPLES}

This section focuses on industrial applications of NMRI to characterize non-medical and nonbiological liquid-like samples. These applications deal with the imaging of spin density, relaxation times, chemical shifts, and fluid-flow velocities for purposes of material characterization. The most readily observed nuclei are ${ }^{1} \mathrm{H},{ }^{31} \mathrm{P}$, and ${ }^{19} \mathrm{~F}$. Many early applications of NMRI imaging dealt with using spin density mapping to judge the absorption of water into a number of different hydrophilic and hydrophobic polymers. One study imaged the difference of water uptake between two glass-reinforced epoxy resins. (7) The difference between the two resins was that one was cured with an aromatic amine, and the other was cured with an anhydride. Though both gained about $1-2 \%$ in weight, the amine-cured system showed water concentrated in the center of the sample while the water was segregated to the outside of the anhydride-cured epoxy.

Since NMRI is non-invasive and real-time, the water uptake by a polymer can be monitored in situ to judge diffusion rate with spin density mapping. The water uptake by Nylon $6,6(8)$ allowed water diffusional rates to be calculated. The image revealed the marked absorption of water over 28 days of exposure.

Weisenberger and Koenig studied the diffusion of methanol and acetone into polymethylmethacrylate (PMMA).(9,10) The 'H NMR images showed the extent of solvent penetration, which ultimately reached the glassy core of the sample. The relaxation time constants reflected the restriction of solvent molecule mobility in the presence of restricted polymer segmental motion at the rubber/glass interface. The studies also showed that the rates of solvent desorption from the polymer were inconsistent across the diameter of the sample.

Webb and Hall studied the penetration of acetone into vulcanized rubber with ${ }^{1} \mathrm{H} N M R I$ to show its practical application. (11) They later examined the absorption of benzene-acetone and isooctane-cyclohexane mixes into vulcanized rubber, taking advantage of both chemical shift selection and multiple quantum coherence.(12)

$T_{1 H^{-}}$and $T_{2 H^{-}}$weighted imagings are common in NMRI. Both are based on properties of the Halon spin-echo sequence. For $T_{2 H}$-weighted imaging, the amplitude of the spin echo decays as a function of $T_{2 H}$. By adjusting the echo delays to be comparable to the $T_{2 H}$ valve of the sample, those structures with long $T_{2 H}$ will be imaged. The signal from components with short $T_{2 \mathrm{H}}$ vanishes. The grey scale of the image thus follows the order of $T_{2 H}$ : long $T_{2 H}$ species display high intensity, and short $T_{2 H}$ species how low intensity (or none at all). In $\mathrm{T}_{2 \mathrm{H}^{-}}$ weighted imaging, the time between $90^{\circ} \times$ pulses, referred to or $T_{F}$ in the medical lecture, must be long compared to $T_{1 H}$ values so that the equilibrium magnetization is re-established.

$T_{1 H^{-}}$-weighted imaging relies on adjusting $T_{R}$ values to be short compared to average sample $T_{1 H} S$ as that certain species with long $T_{1 H}$ become saturated. Species with short $T_{1 H}$ are thus preferentially imaged and show highest signal intensity. Consequently, long $T_{1 H}$ species show low signal intensity (or none at all). $\mathrm{T}_{1 H^{-}}$wighted imaging requires the shortest prossible echo delays to minimize the effects of spin-spin relaxation. 
Another type of $T_{1 H}$ imaging referred to as invena-recovery (IR) images, takes advantage of the $180^{\circ} \times-T-90 x$ pulse sequence for measuring $T_{I_{H}}$ values to selectively image protons with known $\mathrm{T}_{1 H}$ valves. The initial $180^{\circ}$ pulse inverts all magnetization; at short $\mathrm{T}$ valves $(\mathrm{T}$ is the time between the $180^{\circ}$ pulse and the $90^{\circ} \times$ pulse, and is approximately equal to $T_{H H}$ or "time internal," as referred to in the medical literature) protons with long $T_{1 H}$ slow the greatest greyscale intensity. As the extent of long $T$ values, protons with long $T_{1 H}$ show the lowest greyscale intensity.

The mapping of protons with given nuclear relaxation times can be applied to add information about solvent absorption by polymers. For instance, the uptake of toluene by polystyrene(6) showed regions of short- $T_{2 H}$ (interstitially absorbed) toluene and long- $T_{2 H}$ (marginally bound) toluene. The regions possessing long- $T_{2 H}$ toluene were restricted to the outermost layer of the polystyrene, and short- $T_{2 H}$ toluene was restricted to inside the polymer. The swelling and physical degradation of the polystyrene with toluene was monitored in situ. Such systems are important within the DOE weapons complex, since solvent extraction is used to dissolve/swell polymeric binders. Two examples are dimethylsulfoxide use with Viton-A and toluene use with cured silastics. In a similar application, the swelling of a polystyrene specimen exposed to $\mathrm{CCl}_{4}$ was studied(13) with $\mathrm{T}_{1}$-weighted ' $\mathrm{H}$ NMRI imaging and very high strength field gradients.

Rubbers possess sufficient anisotropic molecular motion that they too can be imaged without totally relying on solid-state line narrowing techniques.(8) The images appear to be of somewhat low utility because of poor spatial resolution. For such mobile solids, a chemical shift selective excitation can increase the specificity of the image. Selective excitation makes use of specially shaped long $180^{\circ}$ pulses to produce a narrow excitation bandwidth to invert only given chemical species of a sample, and thus specifically image that one. Using this selectivity, Garrido and Mark(14) were able to selectively image the methine protons of polybutadiene in mixtures of polybutadiene and polydimethylsiloxane.

In special cases, copolymers can effectively be studied with conventional NMRI techniques. For instance, if the two components of the copolymer differ markedly in their glass transition temperatures, then variable temperature NMRI (using a variable-temperature probe) can be used to heat the sample temperature up to between the glass transition temperature of the two components. The component with the lower glass transition temperature will possess enough molecular motion so that it can be differentially imaged. Such imaging showed heterogeneity where none could previously be seen.(8) A similar situation arises from the main product of the Pantex Plant, PBX. A PBX actually consists of the crystalline high explosive component (TNT, RDX, HMX, or TATB) and a single plastic binder (such as Viton $A$, Estane 5703-F1, or Kel-F 800). The great diversity in thermal characteristics between these two groups could be exploited to provide a $T_{2}$-weighted image.

Garrido and co-workers have successfully imaged(15) a series of silica-filled polymers using conventional ${ }^{1} \mathrm{H}$ spin imaging and $T_{1 H} / T_{2 H}$ imaging to see air voids and bubbles within the polymer. Their images showed the presence of dark regions representing the air voids. The authors claimed a digital image resolution of 65 to $200 \mu \mathrm{m}$. Here at the Pantex Plant, the DOE uses extrudable and paste explosives to which Cab-O-Sil (a silica filler) has been added to customize flow characteristics. Perhaps study of these explosives could reveal air voids causing possible failure of the system. 
Spin density and $T_{2 H}$ imaging of geological samples revealed the distribution of porous layers and fine geological structure in a Berea sandstone previously thought to be uniform. (8) Image spatial resolution in this case was $1 \mathrm{~mm}$. Long- $T_{2 H}$ water regions may be consistent with large pore sizes in the sandstone. Conventional NMRI imaging without special line-narrowing techniques can be used to monitor the oil distribution within solid geological samples.(16)

\section{NMRI STUDIES OF CHEMICAL REACTIONS AND PROCESSES}

Apart from material characterization, NMRI has been used to actually study chemistry. Chingas and co-workers(17) have studied the intercalation of AsF5 into the sheet-like structure of graphite through the use of ${ }^{19} \mathrm{~F}$ NMR spin imaging.

Oscillating chemical reactions, which have been reviewed by Scott(18) and Rabai, Orban, and Epstein(19), also provide a stage to demonstrate on unusual imaging ability of 'H NMRI. For instance, the $\mathrm{Mn}^{2+}$ catalyzed Belousov-Zhabotinskii reaction displays in homogeneities in the $\mathrm{T}_{2 H}$ of water, depending upon whether $\mathrm{Mn}^{2+}$ or $\mathrm{Mn}^{3+}$ is nearby. $\mathrm{T}_{2}$-weighted images(20) taken every 20 seconds during the oscillating reaction, depict chemical waved propagating through the reaction vessel. The chemical waves can be reconstructed in 3-dimensions with NMRI.

${ }^{23} \mathrm{Na}$ imaging was capable of showing impact-created defect sites (21) within a $\mathrm{NaCl}$-containing stimulant propellant. The sodium content was markedly lower at the point of impact. Suits and Lutz claimed a spatial resolution of $1 \mathrm{~mm}$. Suits and White, examining a piece of $\beta$ alumina dipped in molten $\mathrm{KNO}_{3}$, were able to see a discontinuity in $\mathrm{Na}$ content at a hairline crack.(22) From this, they foresaw the use of NMRI in detecting cracks. Since the work performed by Suits' group had as one goal the improvement in image quality from simple inorganic solids containing quadrupolar nuclei, they used their newly improved methods to image ${ }^{27} \mathrm{Al}$ signals from the aluminum particles in stimulant propellant. The image of the propellant showed approximately $1-\mathrm{mm}$ spatial resolution. Aluminum is important in propellants and explosives, for it is frequently added to improve the explosive's efficiency. Within the DOE weapons complex, aluminum powder is used as a heat source for certain pyrotechnics such as the thermite $\mathrm{Al} / \mathrm{Fe}_{2} \mathrm{O}_{3}$. Suits and White(23) used ${ }^{81} \mathrm{Br} \mathrm{NMRI}$ to monitor temperature gradients within a sample of $\mathrm{KBr}$ of cubic symmetry.

The curing of epoxy resins has been followed in situ.(24) Solid-state line narrowing techniques were required for this particular case, though in most cases, the time dependence of polymer curing from a liquid or mobile material to a solid can be followed without them. The initial polymerization of methyl methacrylate in the liquid phase has been followed using two- and three-dimensional NMRI.(25)

The reaction of ammonia with substituted benzoic acids was monitored using ' $H$ NMRI and the coherent line-narrowing pulse sequence CMG-48.(26) This reaction was supposed to be enhanced on a given lattice arrangement of a crystal face. The authors used a solid sample whose face they believed to be the correct crystal lattice. The authors found that they could not see a clear-cut enhancement in reaction on the crystal face. They did see scattered areas of enhanced reaction across the crystal surface, which they assigned to inconsistent 
crystalline structure across the crystal face. Enough data were obtained to get an image of some detail and ammonia diffusion information.

\section{NMRI OF SOLID SAMPLES}

So far, this review has addressed liquid samples, liquid-like samples, or samples that could be made to act more liquid-like by increasing the sample temperature or adsorbing the material of interest into a porous solid matrix. Indeed, such an approach tries to study every possible application of conventional NMRI and pushes conventional NMRI to its limit. But where does the realm of conventional NMRI end and the more sophisticated instrumentation and pulse programming of solid-state NMRI/NMR begin? The answer to this question, again, is entirely sample-related. There must be sufficient motion of the molecules in question to create nuclear relaxation times $\left(T_{1}\right.$ or $\left.T_{2}\right)$ long enough to give natural linewidths of tens of $\mathrm{Hertz}(\mathrm{Hz})$ (with conventionally chosen gradient strengths). If this is not the case, then the NMR image will be extensively blurred unless some type of solid-state line narrowing technique is used to initially mimic the isotropic motion of molecules in the liquid state. Line narrowing can be combined with higher gradient field strengths to theoretically produce images of solid samples. These experiments can be performed on conventional solid-state NMR spectrometers, equipped with accessories to provide field gradierits, imaging, and pulse programming. But with all these complications and increasing complexity of the experiment, what continues to motivate the NMRI of solids? As pointed out by Jezzard(2), solid-state imaging can a) provide a means of morphological study of polymer blends and composites, b) theoretically allow any microscopic/macroscopic NMR-detectable property to be imaged, and c) reveal information on aggregation and blending of highly complex solids in a non-invasive way. The high explosive blends and other composite materials used in the DOE weapons complex need such an examination so that the properties of the material in its native, solid state are revealed instead of performing solvent digestion/dissolution prior to characterization. Such sample treatment may destroy the very information we require. To understand the line narrowing techniques that are available, a brief introduction to solid-state line broadening mechanisms follows.

Line broadening is caused from one or more mechanisms. The most important, which is averaged to zero by rapid isotropic molecular motion, is the dipole-dipole interaction. For most organic systems, the most important dipole-dipole interactions are the ${ }^{1} \mathrm{H}-{ }^{1} \mathrm{H}$ and the ${ }^{1} \mathrm{H}-{ }^{13} \mathrm{C}$ dipolar interactions. Their magnitudes are approximately 50 and 20 kilohertz $(\mathrm{kHz})$, respectively. This compares to the more widely known indirect or J-coupling, whose magnitude is from approximately 1 to $270 \mathrm{~Hz}$. In solution, molecules and sections of molecules move much faster than this; by doing so, the direct dipole-dipole interactions are averaged to zero. This is not so with solid samples, and a single peak may be split into a large number of multiplets, the size and magnitude of which is dependent on the total number of dipolar coupled nuclei, the angle between them and the external field, and the inverse of the distance between them cubed. 
The indirect dipolar interaction or J-coupling is present in the solid state as it is in the liquid state. Its magnitude is insignificant compared to the direct interaction. The techniques, therefore, which average the larger dipolar interactions to zero most certainly cause the smaller interactions to disappear.

Another complicating factor is a mechanism called the chemical shift anisotropy. This mechanism, which scales in magnitude directly with field strength, is averaged to its isotropic value (the chemical shift) in solution. In a solid sample, the chemical shift of a given nucleus depends upon the orientation that the particular nucleus' host molecule lies with respect to the external magnetic field. Each orientation gives rise to a unique electronic environment. In a crystalline solid, where all orientations with respect to the external field are possible, the resonances for all orientations of a given nuclei then lie within a given chemical shift range (i.e., the chemical shift anisotropy). The total value of the chemical shift anisotropy may be several tens of $\mathrm{kHz}$.

The final complicating line-broadening mechanism is the quadrupolar interaction. For half integral spins whose $I \neq 1 / 2$, a non-spherical charge distribution arises at the nucleus. The non-spherical charge density gives rise to an electric quadrupole moment which couples with electric field gradients. In electronic environments of high symmetry, there are no electric field gradients and thus no quadrupole interaction. However, such symmetry is rarely the case, so for most quadrupolar nuclei the quadrupolar interaction exists. We assume a "high field" case, where the magnitude of the Zeeman interaction predominates and controls the magnitude of the energy gap between the $1 / 2 \leftrightarrow-1 / 2$ states. The quadrupolar interaction slightly raises or lowers their energy level and thus the resonance frequency. The frequency change is angularly dependent, and a powdered sample gives rise to all possible angles with respect to the external field. The range of resulting frequencies gives rise to broad lines for quadrupolar nuclei; however, imaging of quadrupolar nuclei are not as prevalent as $1=1 / 2$ nuclei. Some examples of imaging were mentioned earlier in this review.(21-23)

Electronic and mechanical means can be used to minimize the line broadening caused by the above interactions. The ${ }^{1} \mathrm{H}-{ }^{13} \mathrm{C}$ dipolar interaction land its smaller indirect coupling counterpart) can be averaged to zero through the use of high-power proton decoupling. Highpower proton decoupling, of course, works well for the observation of ${ }^{13} \mathrm{C}$ nuclei(27), but could not be used for ${ }^{1} \mathrm{H}$ imaging since one would be equalizing the population between the ' $\mathrm{H}$ spin states, thus eliminating the signal.

For eliminating the ${ }^{1} \mathrm{H}-{ }^{-} \mathrm{H}$ dipolar interaction, an examination of the Hamiltonian governing the interaction reveals another term which can be exploited. The Hamiltonian contain: both a geometric part and a spin-space term. Application of "coherent signal averaging techniques," another name for a specific multiple-pulse sequence, moves the macroscopic magnetization through states that when summed over a cycle of pulses renders the dipolar Hamiltonian (to zeroth order) to zero. The first such multiple-pulse sequence was named WAHUHA for its creators, Waugh, Huber, and Haeberlin.(4) More efficient pulse sequences have been developed such as the MREV-8(28), BR-24(29), and CMG-48(30)(vide supra). When used properly, these sequences average the dipolar interaction to zero and yield linewidths that can be profitably imaged. 
Since the Hamiltonian for the dipolar interaction also contains a geometric part, mechanical means can be used to minimize the resulting linewidths. The geometric term, $\left(3 \cos ^{2} \beta-1\right)$, implies that when the sample is rotated at the angle $\beta$ with respect to the external field such that $\left(3 \cos ^{2} \beta-1\right)=0$, the dipolar Hamiltonian will be averaged to zero. Indeed, if sample rotation is fast enough, then spinning the sample at this "magic angle" $\left(54^{\circ} 44^{\prime}\right)$ will satisfy the condition.

However, the magnitude of the dipolar interaction for most samples prevents practical use of magic-angle spinning (MAS) to eliminate the interaction. The equation describing the chemical shift anisotropy contains the same geometric term; the magnitude of this line-broadening mechanism is ideal for treatment by MAS. MAS averages the chemical shift anistropy to its is utropic value and yields linewidths which are reasonably manageable for imaging. Use of this line-narrowing technique with imaging requires that the magnetic field gradient also rotate with the same period as that of the sample rotation to maintain a constant field strength at any one position within the sample. Different slices of the sample for imaging are selected by changing the phase relationship between the sample rotation and the synchronously rotating magnetic field gradient. The slices are then combined to yield the image.

MAS has been used alone to image samples that display small ' $\mathrm{H}-{ }^{1} \mathrm{H}$ dipolar interaction and chemical shift anisotropy. For instance, a small piece of polyisoprene with five holes drilled into its length (31) were imaged in this manner. The results, showing the outer two holes closed off, emphasize the distortional effects of MAS. In this same work, the authors contrast the images of a mechanically blend fraction of polybutadiene-polystyrene with a toluene cast of the same nominal composition. The images show the two blends to be incompatible.

The discussion above focused to a large degree on eliminating the ${ }^{1} \mathrm{H}^{1} \mathrm{H}$ dipolar interaction since ${ }^{1} H$ is the nucleus most widely used as a probe in NMRI. Of course, other nuclei can be imaged. A major drawback with some nuclei is their low natural abundance. ${ }^{13} \mathrm{C}$ imaging is hindered by its $1.1 \%$ natural abundance, though the instrumentation is much improved over that used with ${ }^{1} \mathrm{H}$ NMRI. The signal from the ${ }^{13} \mathrm{C}$ can be enhanced by using cross polarization(32) to transfer magnetization from an abundant nuclear species (i.e., protons) to the ${ }^{13} \mathrm{C}$ nuclei. An added benefit is that the experiments can be repeated on the time frame of the $T_{1 H}$ (relatively short), not the $T_{1 C}$ (potentially longer).

Individual line-narrowing techniques can be used alone or combined to increase efficiency of line narrowing and obtain higher resolution images. For instance, a multiple-pulse linenarrowing sequence like MREV-8 can be used with the conventional NMRI imaging routines to produce images of solids with better spatial resolution than with the conventional routines alone. Likewise, MREV-8 or its improved descendants can be combined with MAS to eliminate both the ${ }^{1} \mathrm{H}-{ }^{9} \mathrm{H}$ dipolar interaction and the ${ }^{1} \mathrm{H}$ chemical shift anisotropy to permit solid-state 'H NMRI. This combination, referred to as Combined Rotation and Multiple Pulse Spectroscopy (or, more appropriately, CRAMPS), has displayed its imaging capability $(33,34)$ by reducing the sample's linewidth from $20 \mathrm{kHz}$ to $100 \mathrm{~Hz}$, resulting in a spatial resolution of $300 \mu \mathrm{m}$. Veeman and Cory (31), however, reported that the magnetic gradient rotating synchronously with the sample reduces the effectiveness of multiple pulse sequences like MREV-8 in averaging the ' $\mathrm{H}-{ }^{\prime} \mathrm{H}$ dipolar interaction. They argue that when MAS alone can average the ${ }^{1} \mathrm{H}^{-1} \mathrm{H}$ interaction, then the multiple-pulse option should be dropped. 
Insensitive nuclei such as ${ }^{13} \mathrm{C}$ can be imaged by combining MAS with cross polarization (CP/MAS) to enhance the signal from the ${ }^{13} \mathrm{C}$ nuclei. The authors of this imaging technique(27) obtained a 1-dimensional image of a $6-\mathrm{mm}$ ring of adamantine with an optimal spatial resolution of $110 \mu \mathrm{m}$. Given the array of different nuclei whose signal could be enhanced by cross polarization from protons and the image selectivity imparted by selecting different nuclei, this technique appears to particularly promising.

\section{CONCLUSION}

Though the wide variety of NMRI techniques available for use appears at first somewhat baffling, upon closer examination the choice of imaging technique is very sample dependent. For instance, conventional NMR images can image a wide variety of samples, ranging from mostly biological samples and water-impregnated solid matrices to highly mobile elastomers. In fact, the ability to image water as it penetrates into a sample gives in situ data on molecular diffusion and sample compatibility. Since the techniques can be extended to image other solvents, the resilience of polymers to a variety of solvents can lead to correct identification of combinations of compatible materials. Variable-temperature techniques broaden the number of samples which should yield useful magnetic resonance images. This statement is especially true when sample components differ greatly in temperature-related motion.

Methods are now available for the magnetic resonance imaging of rigid solids by applying the line-narrowing and signal-enhancing methods developed for solid-state NMR. Fairly remarkable spatial resolution has been achieved for both ${ }^{1} \mathrm{H}$ and ${ }^{13} \mathrm{C}$ imaging, and approaches the limits established by $X$-ray imaging. In some cases, the resolution may provide better or complementary data not presently available.

A number of possible applications with weapon materials have been presented during the course of this report. Many more come to mind. Some of these are:

- Imaging of ${ }^{11} \mathrm{~B}$ and ${ }^{29} \mathrm{~S}$; in boron-loaded polydimethylsiloxane

- ${ }^{\prime} \mathrm{H}$ imaging of the curing of different siloxanes

- Following the initial cross-linking of anide-used epoxy resins

- Studing the resistance of PBXs and polymer weapon components to attach by solvents such as d-limonenene

- Examining potting materials and extrudable explosives for voids

- Determining the degree of attach of initiated, fluorinated organics on plastics

- Studing the long-term effects on PBX binders and other polyenic materials of low-level, long-term $y$ radiation, neatness, and tritium

- Imaging of latex-based explosives to guide its development 
The imaging capability, the chemical specificity of NMRI, and the wide variety of possible applications merit the establishment of a cooperative imaging program with a vendor or national lab to realize NMRl's true value to the DOE Complex. 


\section{REFERENCES}

1. P. Mansfield and P. G. Morris, Advances in Magnetic Resonance, Suppl. 2. NMR Imaging in Biomedicine, J. S. Waugh, Ed.; Academic Press: New York, 1992.

2. P. Jezzard, J. J. Attard, T. A. Carpenter, and L. D. Hall, Prog. in NMR Spectro., 1991, $23,1-41$.

3. C. S. Yannoni, Acc. Chem. Res., 1982, 15, 201-208.

4. J. S. Waugh, L. M. Huber, and U. Haeberlen, Phys. Rev. Lett., 1968, 20, 180.

5. (a) I. J. Lowe, Phys. Rev. Lett., 1959, 2, 285; (b) E. R. Andrew, Arch. Sci. (Geneva), 1959, 12, 10s; (c) E. R. Andrew, Prog. Nucl. Magn. Reson. Spectrosc., 1971, 8, 1.

6. W. P. Rothwell and P. P. Gentempo, Bruker Reports, Vol. 1/1985 (available from Bruker Instruments Inc., Manning Park, Billerica, Ma., 01821).

7. W. P. Rothwell, D. R. Holecek, and J. A. Kershaw, Sci. Polym. Lett., Ed. 1984, 22, 241.

8. W. P. Rothwell, P. N. Tutunjian, and H. J. Vinegar, Proceedings of the Third Symposium of the Industry-University Cooperative Chemistry Program of the Department of Chemistry. Texas A\&M University, March 31 - April 3, 1985. (B. L. Shapiro, Ed., Texas A\&M University Press, College Station, Texas).

9. L. A. Weisenberger and J. L. Koenig, Macromolecules, 1990, 23, 2454-2459.

10. L. A. Weisenberger and J. L. Koenig, Macromolecules, 1990, 23, 2445-2453.

11. A. G. Webb and L. D. Hall, Polym. Commun., 1990, 31, 422-425.

12. A. G. Webb and L. D. Hall, Polym. Commun., 1990, 31, 425-427.

13. M. Corti, F. Borsa, and A. Rigamonti, J. Magn. Reson., 1988, 79, 21-27.

14. L. Garrido and J. E. Mark, Poly. Prepr. (Am. Chem. Soc., Div. Polym. Chem.), 1989, 30, 217-218.

15. L. Garrido, J. L. Ackerman, C. Chang, and J. E. Mark, Meeting of the Division of Polymer Chemistry, American Chemical Society, 1990, 31, 147-148.

16. L. W. Jelinski, Nuclear Magnetic Resonance Spectroscopy, (F. A. Bovey, L. W. Jelinski, and P. A. Mirau, Eds., Academic Press, New York). 1988. p.505. 
17. G. C. Chingas, J. Milliken, H. A. Resing, T. Tsang, and T. Synth. Met. 1985, 12, 131136.

18. S. K. Scott, Acc. Chem. Res., 1987, 20, 186-191.

19. G. Ra'bai, M. Orba'n, and I. R. Epstein, Acc. Chem. Res., 1990, 23, 258-263.

20. A. Tzalmona, R. L. Armstrong, M. Menzinger, A. Cross, amd C. Lemaire, Chem. Phys. Lett., 1990, 174, 199-202.

21. B. H. Suits and J. L. Lutz, Appl. Phys., 1989, 65, 3723-3730.

22. B. H. Suits and D. White, Sol. State Commun., 1984, 50, 291-295.

23. B. H. Suits and D. White, J.Appl. Phys., 1986, 60, 3772-3773.

24. J. B. Miller, unpublished results reported in JACS, 1992, 114, 125-135.

25. P. Jackson, N. J. Clayden, N. J. Walton, T. A. Carpenter, L. D. Hall, P. Jezzard, and C. Wiggins, 10th EENC; Veldhoven, The Netherlands, May 28 - June 1, 1990, Abstract C.6.

26. L. G. Butler, D. G. Cory, K. M. Dooley, J. B. Miller, A. N. Garroway, J. Am. Chem., Soc. 1992, 114, 125-135.

27. D. G. Cory, and W. S. Veeman, J. Phys. E: Sci. Instrum., 1989, 22, 180-184.

28. W. K. Rhim, D. D. Elleman, and R. W. Vaughan, J. Chem. Phys., 1973, 58, 1772.

29. D. P. Burum and W. K. Rhim, J.Chem. Phys., 1979, 71, 944.

30. D. G. Cory, J. B. Miller, and A. N. Garroway, J. Magn. Reson., 1990, 90, 205-213.

31. W. S. Veeman and D. G. Cory, Advances in Magnetic Resonance, 1989, 13, 43-56.

32. A. Pines, M. G. Gibby, and J. S. Waugh, J. Chem. Phys. 1977, 66, 361.

33. D. G. Cory, A. M. Reichwein, J. W. M. Van Os, and W. M. Veeman, Chem. Phys. Lett., 1988, 143, 467.

34. D. G. Cory, A. M. Reichwein, J. W. M. Van Os, and W. S. Veeman, Polym. Prepr., $1988,29,92$. 


\section{DISTRIBUTION}

DEPARTMENT OF ENERGY - WASHINGTON, DC

Director of Weapons Production

Office of Military Application

\section{DEPARTMENT OF ENERGY - AL}

Tochnical Information Officer, IPS

Quality Operations Branch Woapons Quality Division

Technology Development Branch Woanons Quality Division

Waste Menagement Branch Waste Management and

Operational Surety Division

Program Support Branch Weapon Programs Division

\section{DEPARTMENT OF ENERGY - AAO}

Area Manager

\section{OFFICE OF SCIENTIFIC AND TECHNICAL} INFORMATION - OAK RIDGE, TN

(114)

\section{LAWRENCE LIVERMORE NATIONAL LABORATORY \\ L-32 \\ L.53 \\ L-125 \\ $L-282(6)$ \\ L-394}

\section{LOS ALAMOS NATIONAL LABORATORY}

$M-1(3)$

$M-7$

$M-D O$

$W X-1$

WX-3 Group Office

NWT/NWP, F-630

ISD-4
SANDIA NATIONAL LABORATORIES - ALBUQUERQUE

Org. 300

Org. 2571

Org. 2512

Org. 5100

SANDIA NATIONAL LABORATORIES - LIVERMORE

Org. 5354

\section{EG\&G MOUND APPLIED TECHNOLOGIES}

Manager, Product and Process Devolopment

\section{SAVANNAH RIVER LABORATORY}

Program Manager, Defense Waste Processing Section, 773-A

\section{ALLIED-SIGNAL INC. - KANSAS CITY PLANT}

Material Engineering

\section{MASON \& HANGER - PANTEX PLANT}

\author{
Operations Directorate \\ Engineering and Design Division \\ ES\&HMM Directorate \\ Explosives Technology Division \\ Manufacturing Division \\ Program Management \\ Quality Division \\ Circulation Copy: \\ 1. R. M. Loghry, 12.69 \\ 2. S. G. Hallett, 11-2 \\ Tochnical Library \\ File (5) \\ Please refer any distribution changes to \\ A. V. Lucero, Publications Section FTS 477-3535.
}


$1 !$
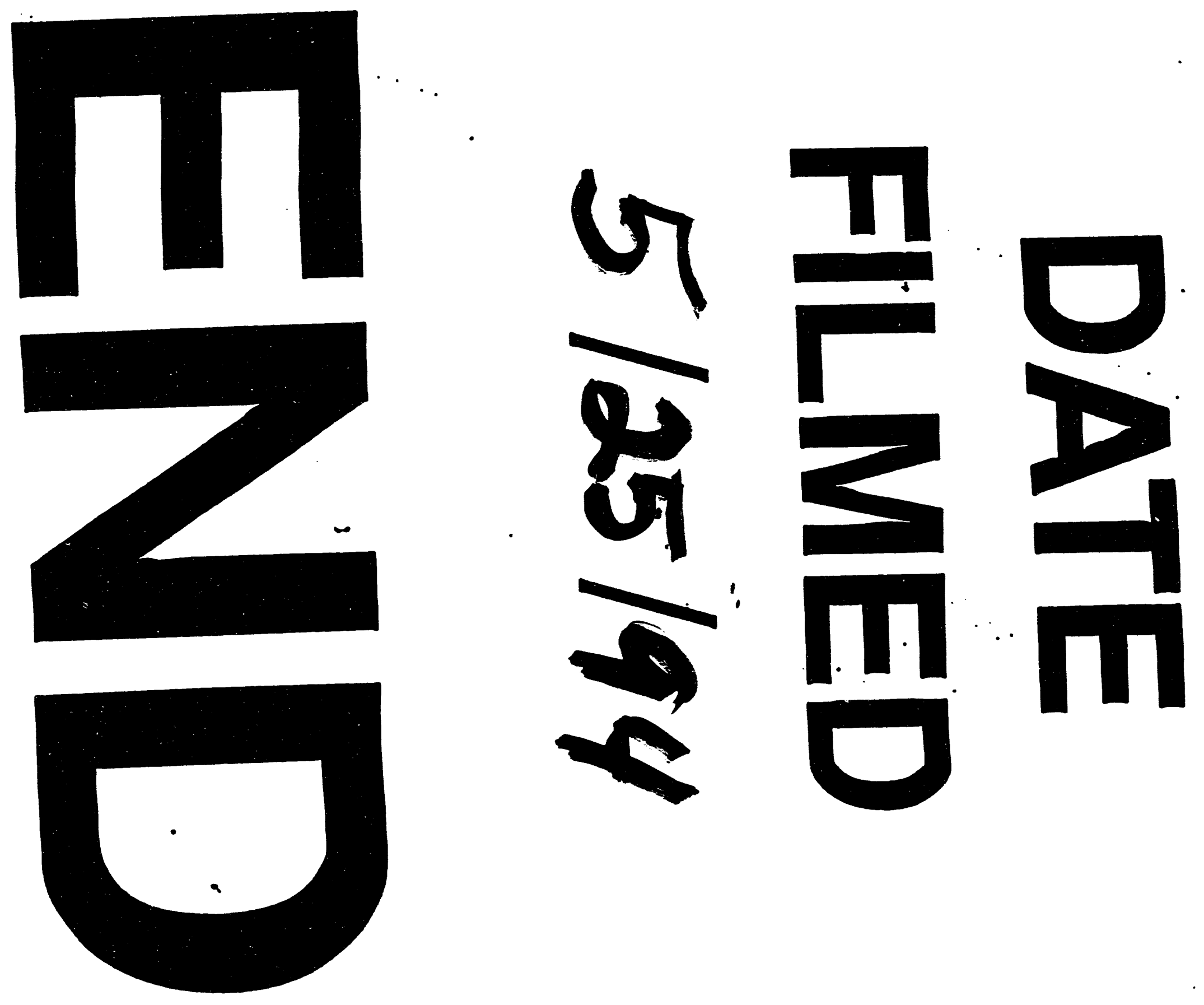


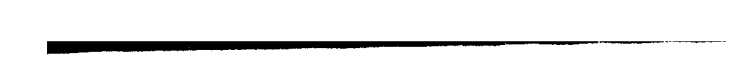

